\title{
Práctica clínica basada en evidencia: porqué y como aplicarla en odontopediatría
}

\section{Resumen}

La práctica en el área de salud tradicionalmente se basan en la experiencia clínica, libros ó estúdios de menor calidad metodológica. El objetivo del presente artículo es demostrar al clínico que atende niños la importancia de buscar la mejor evidencia disponible en fuentes científicas de información. Además se pretende presentar algunas herramientas para que el odontopediatra pueda obtener la mejor evidencia posible y aplicarla en la práctica clínica. Inicialmente se presentan los modelos tradicionales de la práctica clínica: basadas en la opinión de autoridades, el
Carlos Alberto Feldens ${ }^{1}$ Paulo Floriani Kramer ${ }^{2}$

\section{Artigo Original}

\section{Prática clínica baseada em evidências: por que e como aplicar na Odontopediatria}

\section{Resumo}

Práticas na área da saúde tradicionalmente baseiam-se na experiência clínica, livros ou estudos de menor qualidade metodológica. O objetivo do presente artigo é demonstrar ao clínico que atende crianças a importância de buscar a razonamiento fisiopatológico, en los resultados intermedios y la evidencia preliminar. A continuación se definen y ejemplifican la importancia de la práctica clínica basada en evidencia. Una secuencia se presenta para que el clínico pueda ejercer un modelo de práctica basada en evidencias y que incluye: la elaboración de la pregunta que resume la pregunta clínica, la búsqueda bibliográfica y selección de la mejor evidencia científica, y la aplicación de evidencia para la toma de decisiones en la práctica clínica.

Palabras clave: Práctica Clínica Basada en la Evidencia, Odontología Pediátrica, Salud del Niño. 
seadas na opinião de autoridades, no raciocínio fisiopatológico, em desfechos intermediários e evidências preliminares. A seguir é conceituada e exemplificada a importância da prática clínica baseada em evidências. Um roteiro é então apresentado para que o clínico possa exercer um modelo de prática baseada em evidências, o que inclui: elaboração da pergunta que resume a dúvida clínica; busca da literatura e seleção da melhor evidência científica; e aplicação da evidência para a tomada de decisões na prática clínica.

Palavras-chave: Prática Clínica Baseada em Evidências, Odontopediatria, Saúde da Criança.

\section{Original article}

\section{Evidence based clinical practice: why and how to apply it in pediatric dentistry}

\begin{abstract}
Practices in the health field are traditionally based on clinical experience, text-books or studies of low methodological quality. The aim of the present study was to demonstrate to clinicians who treat children the importance of seeking the best evidence available in scientific sources of information. A further aim was to present tools for pediatric dentists to be able to find the best possible evidence and apply it to their clinical practice. Traditional clinical practice models based on the opinion of authorities, physiopathological reasoning, surrogate outcomes and preliminary evidence are first presented. Next, the importance of evidence-based clinical practice is stressed and exemplified. A routine is then outlined to allow clinicians to employ an evidencebased practice model, including a question that summarizes clinical doubts, a search of the literature, the selection of the best scientific evidence and the application of this evidence during decision making in clinical practice.
\end{abstract}

Key words: Evidence-Based Practice, Pediatric Dentistry, Child Health.

\section{Introducción}

Elegir los procedimientos $\mathrm{u}$ orientaciones para la prevención y control de las diferentes afecciones del ámbito de la odontopediatría no pueden, en los tiempos actuales, ser fruto sólo de opinión o experiencia personal. La adopción de prácticas en salud basadas en evidencias representa una forma de proporcionar al paciente estrategias de prevención o tratamiento con mayor posibilidad de traer desenlaces positivos. Las decisiones clínicas pasan a depender fuertemente de investigaciones con buena calidad metodológica y que preferentemente estimen beneficios, riesgos y costos. Además de eso, llevan en consideración su aplicación en diferentes contextos y las preferencias de los pacientes (1-3).

El objetivo de este artículo es contribuir para la comprensión de que las prácticas clínicas en la odontopediatría deben basarse en evidencias científicas. A la vez, se pretende auxiliar al clínico en la búsqueda de información científica de calidad y que contribuya para las diferentes etapas de actuación profesional, del diagnóstico a las conductas preventivas y terapéuticas. 


\section{Práctica clínica basada en evidencias: ¿por qué?}

La historia de la Medicina y Odontología demuestra una gran evolución en el conocimiento desde la época del curanderismo y hechicería. Aún así, fue sólo en las dos últimas décadas del siglo XX que se construyó el consenso, en el ámbito científico, sobre la necesidad de basar la práctica clínica en experimentos metodológicamente bien diseñados. De una forma general, esta conclusión fue motivada a partir de la observación de que los métodos tradicionales no contribuían decisivamente para el diagnóstico, prevención y tratamiento de las diferentes patologías. En otras palabras, las enfermedades eran percibidas tarde demasiado, los enfermos no mejoraban con el tratamiento realizado y nuevos enfermos aparecían. Ni aún la distribución de las afecciones en la población con un nivel mínimo de precisión era conocida. En este contexto, se hace imposible establecer, en el área de la salud, la mejor estrategia de diagnóstico, la terapia más efectiva o lo afectado que debe ser prioritariamente combatido (3).

Un simple ejemplo, en este sentido, es la escasez de datos nacionales confiables en los diferentes países del mundo, con énfasis para América Latina, en relación a la prevalencia de diferentes afecciones en la salud oral. En Brasil, fue sólo en el inicio del siglo XXI que un levantamiento epidemiológico con procedimientos metodológicos adecuados fue llevado a efecto, propiciando el desarrollo de una base de datos que fuera capaz de orientar políticas y acciones con base en el que realmente la población necesita. La respuesta para preguntas simples como: ¿cuánto la caries dental alcanza niños, adolescentes y adultos en la población brasileña?”, “¿cuáles son las necesidades de tratamiento relacionadas a la caries dental?”, “¿cuál es la prevalencia de enfermedad periodontal?”, “ ¿transtornos oclusales representan un problema en Brasil?" o “¿cuál es la necesidad de prótesis en las diferentes edades?".

De una manera general, la práctica clínica actual en las ciencias de la salud, en especial en la Odontopediatría, aún está fuertemente contaminada por la antigua plantilla, cuyos ejemplos principales son la práctica clínica basada en la opinión de la autoridad, en el raciocinio fisiopatológico o en desenlaces intermediarios, ejemplificados a continuación.

\section{Práctica clínica basada en la opinión de la autoridad}

En este modelo, "consensos" son reproducidos, enseñados y practicados con base en la autoridad de quien los establece (3). Con origen en la propia evolución de los cuerpos docentes de las universidades, en que el "un óptimo clínico es un excelente profesor", tales prácticas pueden llevar la conductas innecesarias o hasta iatrogénicas. Como ejemplo se puede citar las afirmaciones, continuamente reproducidas por la "autoridad", de que "explorador que se traba en la fisura oscurecida indica diente cariado..." o que "el tercer molar invariablemente produce apiñamiento dentario". A pesar de que tales "conocimientos" han sido desmentidos por la investigación científica, su aplicación aún persiste en la práctica clínica odontológica. Peor que eso, estos conceptos aún son reproducidos en modelos cuyo enseñanza no evoluciona en consonancia con el conocimiento científico generado. Además de eso, repetidos cíclicamente por el clínico, estos conocimientos son "aprendidos" por los propios pacientes que materializan su preocupación con "un punto negro en el diente" o con "los dientes chuecos a causa de la muela del juicio". No parece necesario profun- 
dizar cuánto tales "conocimientos" conducen a procedimientos innecesarios, gastos y daños a los pacientes.

\section{Práctica clínica basada en el raciocinio fisiopatológico}

Una evolución de la etapa anterior es basar la práctica clínica en el raciocinio fisiopatológico. En este sentido, métodos de diagnóstico y conductas preventivas o terapéuticas son llevados a efecto basados, por ejemplo, en el mecanismo fisiopatológico de las enfermedades. Frecuentemente la utilización de este modelo no conduce a la mejor estrategia de abordaje. Un ejemplo claro en la Medicina es: "la inmovilización postfractura ósea contribuye para la cicatrización de los tejidos, por lo tanto tejidos traumatizados deben ser inmovilizados". Los ensayos clínicos llevados a efecto para confirmar/negar tal hipótesis en el caso de "efecto de látigo" (trauma accidental que afecta la región cervical) demostraron ningún beneficio y hasta perjuicio con la adopción de esta conducta (4). En la Odontología, la observación de los mecanismos que llevan a la fluorosis (consumo de flúor por encima de la dosis crónica potencialmente tóxica) originó la "orientación" de que niños pre-escolares deberían utilizar dentifrico sin flúor o con baja concentración. Aún después de la demostración por medio de ensayos clínicos y revisiones sistemáticas (las mejores estrategias de investigación para verificar el efecto preventivo y terapéutico), la fuerza de la orientación basada en raciocinio fisiopatológico aún viene privando a los niños de una importante estrategia de salud pública. Más que eso, ha representado un cruel mecanismo de desigualdad, pues alcanza quién más necesitaría de su beneficio.

\section{Práctica clínica basada en desenlaces intermediarios}

La adopción de estrategias basadas en estudios que utilizan desenlaces intermediarios puede no traer beneficio o inclusive producir daño a los pacientes. Desenlaces intermediarios, sustitutos o criterios de valoración indirectos (surrogate endpoints) son medidas fisiológicas o laboratoriales utilizadas en estudios como sustitutos de desenlaces clínicamente relevantes para prever los efectos clínicos de una terapia. Aún algunas medidas clínicas, no percibidas por los pacientes como relevantes, se constituyen en desenlaces intermediarios. Ejemplos de ese tipo de desenlace en la odontología son: flujo salivar, capacidad tampón de la saliva, sangramiento gingival, índice de placa visible, consumo de azúcar o frecuencia de cepillado dental. Y ha desenlaces clínicamente relevantes (hard endpoints) son aquellos reconocidos por los pacientes como condiciones que efectivamente impactan en su vida. En la Odontología son representados por caries dental, pérdida dental, dolor y, más modernamente, calidad de vida relacionada a la salud oral.

Hay una serie de ejemplos, en el área de la salud, de que mejora en desenlaces intermediarios no necesariamente implica mejora en desenlaces clínicamente relevantes. Un ejemplo de desenlace intermediario, en la Medicina, es la arritmia ventricular. Como se demostró que este factor está claramente asociado a la muerte súbita, el presupuesto es que la supresión farmacológica de la arritmia ventricular prevendrá la muerte súbita. Sin embargo, el ensayo clínico diseñado para tal tuvo que ser interrumpido por exceso de mortalidad en el grupo que utilizó el fármaco que reduce arritmia (5).

En la Odontología, algunos estudios describieron estrategias que mejoraron la calidad de las 
prácticas de higiene de las familias, disminuyeron el consumo de dulces o redujeron la acumulación de placa bacteriana (6-9). Sin embargo, eso no repercutió en una menor ocurrencia de caries, no asegurando al gestor o al clínico el beneficio de la intervención.

Es importante destacar que el uso de desenlaces intermediarios es fundamental en las fases incipientes de investigación clínica, cuando se está buscando el potencial beneficio y/o seguridad de nuevas intervenciones. Tales estudios son muy importantes para generar hipótesis que van a basar ensayos clínicos con desenlaces clínicamente relevantes. Sin embargo, son inadecuados para basar definitivamente la mejor estrategia de intervención para diferentes afecciones.

\section{Práctica clínica basada en evidencias preliminares}

La producción de evidencias que apoyan el uso de intervenciones pasa por varias etapas, desde las más incipientes, como estudios in vitro, in situ, modelo animal, hasta los más decisivos - experimentos clínicos capaces de expresar el beneficio real a los pacientes. Beneficio de fármacos $\mathrm{u}$ otras intervenciones en estudios preliminares, aunque envuelvan mejora en desenlaces relevantes, no garantizan la efectividad de la intervención. De entrada debe considerarse el diseño del estudio y su calidad metodológica. En este sentido, el beneficio de intervenciones sólo puede ser realmente medido a partir de ensayos clínicos randomizados de buena calidad metodológica, como será discutido adelante. Así, estudios observacionales, aunque consistentemente indiquen beneficio de un comportamiento o fármaco, nunca representan evidencia de su beneficio.
Hay más de 30 estudios observacionales que describen una menor ocurrencia de enfermedad cardiovascular - un desenlace clínicamente relevante - en mujeres que hacían uso de terapia de reposición hormonal después de la menopausia. Sin embargo, el ensayo clínico que probó tal estrategia demostró mayor número de eventos cardiovasculares y cérebrovasculares en mujeres del grupo de intervención (10). La explicación para la aparente incoherencia es más común cuando se compara estudios observacionales con estudios de intervención adecuadamente conducidos: la presencia de factores de confusión. En verdad, las mujeres que hacían uso de terapia de reposición hormonal en los estudios observacionales realmente presentaban más pequeña frecuencia de enfermedad cardiovascular. Sin embargo, lo que explicaba tal relación eran otros factores comunes a aquellas que usaban la terapia, como mayor búsqueda de servicios de salud o mayor auto-cuidado. Tales condiciones, conocidas como "factores de confusión", estaban “escondidas" por detrás del uso de la terapia.

En la Odontología, de la misma forma, el hecho de observarse menor incidencia de caries en niños de una pre-escuela en que hay un programa supervisado de cepillado dental, cuando comparadas con niños de otra institución sin tal programa, no representa evidencia de beneficio del programa. Es posible que niños de la primera escuela puedan diferir en relación a otras características, como nivel socioeconómico o acceso frecuente a alimentos dulces y que estos "factores de confusión" son de hecho los responsables por la diferencia relatada (11). Así, y conforme será discutido adelante, el clínico necesita estar atento a la jerarquía de los estudios en relación a su poder de suministrar la mejor evidencia.

Este breve análisis conduce inevitablemente al cuestionamiento sobre la mejor salida frente a 
tales situaciones. Mantenerse en la "zona de comodidad" y reproducir el conocimiento basado en una plantilla nítidamente ultrapasada o investigar nuevos saberes? Parece no haber más dudas de que las prácticas clínicas basadas en una plantilla tradicional no trajeron mejora en desenlaces relevantes para los pacientes. En el que, entonces, basar la práctica clínica? Lo que la investigación tiene a ver con eso?

\section{Práctica clínica basada en evidencias}

A partir del que fue abordado anteriormente, se deduce que las investigaciones conducidas en animales o en fragmentos de tejidos o células de seres humanos o los estudios observacionales en seres humanos no responden cuestiones clínicas como “¿cuál el mejor tratamiento para este problema?".

El gran número de intervenciones, cada una con potenciales de beneficio, riesgo y costo diferenciados, exigen respuestas más seguras, basadas en investigaciones sistematizadas (investigación) sobre cuestiones clínicas específicas. Al contrario de la experiencia profesional (investigación no-sistematizada), esas investigaciones requieren rigor metodológico que control sesgos característicos del contexto clínico de investigación. Eso incluye objetivos e hipótesis definidos a priori; estandarización de medidas y definición de desenlaces clínicos; y expresión cuantitativa de los beneficios, riesgos y costes asociados su aplicación clínica (1-3).

La práctica clínica basada en evidencias en el área de la salud es el uso consciente, explícito y juicioso de las mejores evidencias actuales disponibles para la toma de decisiones acerca del cuidado de los pacientes (2). Adoptar tal plantilla exige superar la fase de prácticas basadas en la opinión de clínicos, ponentes de congre- sos o autores de libros-texto (3). Además de eso, transciende la experiencia clínica o la simple comprensión sobre la fisiopatología de la enfermedad. Las decisiones clínicas pasan a depender fuertemente de investigaciones con buena calidad metodológica y que preferiblemente estimen beneficios, riesgos y costes.

Tales investigaciones, envolviendo concomitantemente el área básica y aplicada, cada una a su tiempo y en consonancia con evoluciones propias en cada área, han representado un aumento sustancial de innovaciones en salud. Productos de las industrias de materiales y diagnósticas, avances en el conocimiento sobre conductas preventivas o la mejor terapia demandaron investigaciones clínicas con criterios para establecer la magnitud de los beneficios, de los riesgos y de los costes asociados su aplicación en el cuidado de los pacientes (3). Adoptar la práctica clínica basada en evidencias representa un gran desafío para las diversas áreas de la salud, propiciando real beneficio para el paciente y población y evitando gastos innecesarios para los diferentes sistemas de salud.

La adopción de esta plantilla viene produciendo cambios en la Medicina desde el final del siglo pasado, con una parte creciente de las conductas pasando a basarse en evidencias adecuadas, con énfasis para ensayos clínicos randomizados (12). En Odontología, sin embargo, son incipientes las investigaciones que contribuyen para prácticas científicamente correctas, con una parte considerable de las conductas aún basándose en la plantilla antigua (13). Además de eso, una cuota importante del conocimiento generado no es aplicada, permaneciendo un largo camino entre la investigación científica y la práctica clínica. Ambas circunstancias demuestran la oportunidad de los profesionales comprometidos con la producción del conocimiento científico contri- 
buyan para que la población - razón de ser de la propia ciencia - sea beneficiada pelo que se produce.

\section{Práctica clínica basada en evidencias: ¿Cómo?}

Ejercer una modelo de práctica clínica basada en evidencias envuelve, didácticamente, tres fases distinguidas: (1) Elaborar una pregunta que resuma la duda clínica; (2) Buscar la mejor evidencia científica disponible en relación a la pregunta; y (3) Interpretar y aplicar la evidencia para tomar la conducta más adecuada $(1,3,14,15)$. Estas fases(o etapas) son abordadas a continuación.

\section{Elaborar una pregunta que resuma la duda clínica}

Aunque tradicionalmente el deseo de actualización o a busca por novedades pueda envolver temas amplios (como p.ej. "caries en la dentición primaria"), en la mayoría de las veces los clínicos buscan la respuesta para cuestiones específicas de la práctica clínica. Transformar una duda clínica en "pregunta adecuada" representa la primera fase de la práctica basada en evidencias (14). Para facilitar la construcción de la pregunta, es recomendable que la misma incluya:

a) Las características de la población (p.ej.: bebés o pre-escolares o adolescentes o pacientes en fase de la dentición mixta).

b) La exposición considerada (se está evaluándose un factor de riesgo) o a(s) intervención(nes) (si objetivo es evaluar una estrategia preventiva o terapéutica).

c) El desenlace o condición de salud (p.ej.: caries, maloclusión, traumatismo dental).
Ejemplos de preguntas adecuadamente construidas en relación a factor de riesgo e intervención serían, por ejemplo:

- Pregunta (factor de riesgo): ¿Biberón nocturno es factor de riesgo para caries en la infancia?

(En este caso: población = infancia; exposición = biberón nocturno; desenlace + caries).

- Pregunta (intervención): Sellador es más efectivo que barniz fluorado para prevención de lesiones de caries oclusales en primeros molares permanentes recién erupcionados?

(En este caso: población=pacientes de aproximadamente 6 a 9 años; intervenciones $=$ selladores y barniz fluorado; desenlace=caries).

La construcción adecuada de la pregunta que represente la duda clínica contribuye mucho en la búsqueda de artículos que consigan responderla. A partir de la pregunta, se sobreentiende el objetivo a ser perseguido en la búsqueda de la mejor evidencia científica. En los casos anteriormente ejemplificados los objetivos serían:

- Objetivo (factor de riesgo): investigar si biberón nocturno es factor de riesgo para la ocurrencia de caries en la infancia

- Objetivo (intervención): comparar la efectividad de selladores y barniz fluorado en la prevención/control de caries en primeros molares permanentes recién irrumpidos.

\section{Busca de la literatura y selección de la mejor evidencia científica}

Una búsqueda adecuada (correcta) de la mejor evidencia producida es una etapa fundamental, 
pues definirá si la conducta tiene base en la literatura consultada de forma sistemática (y por lo tanto con mayor credibilidad científica) o en literatura de preferencia personal del clínico (constituyendo conocimiento limitado). Las evidencias que fundamentan las mejores conductas clínicas se basan en artículos originales, revisiones sistemáticas o directrices, desde que construidas respetando la jerarquía de evidencias y contengan el grado de recomendación $(3,15)$. Sigue abajo una breve conceptualización de cada una de estas fuentes de consulta y algunas webs sugeridas para búsqueda de evidencias.

\section{Artículos originales}

Los artículos originales relatan los resultados de una investigación y se constituyen en la principal fuente de consulta sobre cuestiones clínicas. El número extremadamente alto de publicaciones cada semana o mes, asociado a la mala calidad de muchas publicaciones determinan la necesidad del clínico conducir adecuadamente la búsqueda y selección de los artículos, lo que será abordado adelante. Entre las fuentes principales para búsqueda de artículos originales se destacan:

- MEDLINE (http: / / www.ncbi.nlm.nih.gov/ pubmed/).

- $\quad$ LILACS (http:/ / lilacs.bvsalud.org/).

- SCIELO (http://www.scielo.org/php/index.php).

\section{Revisiones Sistemáticas}

Revisiones Sistemáticas (RS) representan un tipo específico de artículo original en que se pretende responder a alguna pregunta específica sobre tratamiento, factor de riesgo o pronóstico.
Son así designadas por aplicar técnicas específicas y explícitas de identificación de investigaciones originales y de abstracción de datos en la literatura. En otras palabras, es realizada una selección rigurosa de estudios con óptima calidad metodológica, siguiendo un plan explícito paso a paso y establecido a priori. Con eso se minimiza la posibilidad de sesgos distorsionen los resultados. Después de analizar los resultados de los estudios seleccionados, los autores normalmente expresan sus conclusiones en relación a la pregunta que generó la investigación.

Dependiendo de las características de los estudios obtenidos por la búsqueda, las medidas de efecto (que contabilicen el efecto de un factor de riesgo o intervención) de diferentes estudios pueden ser resumidas en una única medida de efecto, caracterizando el "metaanálisis".

Entre las fuentes para la investigación de revisiones sistemáticas y metaanálisis se destaca:

- Biblioteca Cochrane: http:// cochrane.bireme.br /

\section{Directrices Clínicas}

Directrices en el área de la salud representan recomendaciones a los clínicos sobre conductas específicas de la práctica profesional que se basan en la mejor evidencia científica disponible. Directrices de alta calidad representan una aplicación adecuada de las evidencias de investigación a la realidad de la práctica clínica. De una manera general, se reconoce que no es posible para el clínico, aún con un gran esfuerzo, encontrar por sí sólo todos los artículos esenciales para su práctica clínica. Así, él delega la tarea para un "intermediario de confianza", que irá a revisar muchos estudios y seleccionar los artículos en consonancia con criterios pre-establecidos y 
reconocidos internacionalmente. Las siguientes fuentes pueden ser investigadas en la búsqueda de directrices:

- US National Guideline Clearinghouse: http://www.guidelines.gov/.

- UK National Eletronic Library sea Health: http:/ / www.library.nhs.uk/default.aspx.

La selección de la "mejor evidencia disponible", independiente de derivar de artículo original, revisión sistemática o directriz, es un proceso complejo y que sobreentiende la comprensión de algunos conceptos básicos. Entre estos, merecen ser destacados dos principales, que contribuyen en la "jerarquización" de la mejor evidencia: (1) Diseño del estudio, con valorización del rigor metodológico; (2) Tipo de desenlace.

El grado de generar evidencias en cuanto a conductas clínicas de una Revisión Sistemática es completamente superior a un estudio de cohorte aislado $(16,17)$. Así, el clínico debe reconocer la siguiente jerarquía (decreciente en relación al poder de evidencia científica): revisiones siste-

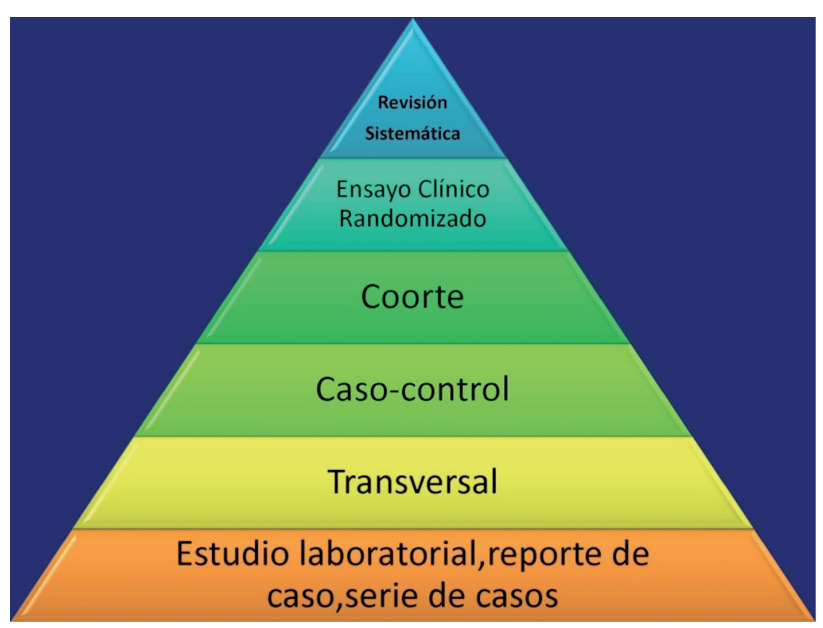

Figura 1. Jerarquía de evidencias de artículos científicos (Adaptado de: Oxford-Centre for Evidence Based Medicine. Revisiones sistemáticas Ensayos clínicos randomizados Cohorte Transversales Estúdios laboratoriales, relatos de casos, serie de casos). máticas, ensayos clínicos randomizados, estudios de cohorte, estudios de casos y controles, estudios transversales y serie de casos (Figura 1).

Ejemplificando, el hecho de un grupo de niños sometidos a algún procedimiento preventivo presentar meses o años después de una baja incidencia de caries (serie de casos), no significa que el procedimiento usado contribuye para el desenlace. De lo contrario, un procedimiento aplicado en un grupo de niños definido a priori $\mathrm{y}$ de forma completamente aleatoria (randomización) que produjo menos incidencia de caries comparado a un grupo control también definido aleatoriamente (ensayo clínico randomizado), presenta una alta probabilidad de ser efectivo.

Además del diseño en sí, cuestiones de validez del estudio deben ser analizadas. En relación al ejemplo arriba, es muy importante verificar si la randomización produjo grupos verdaderamente homogéneos, de forma a poderse atribuir las diferencias en la incidencia de caries a la intervención. Entre varios otros aspectos relacionados a la validez, debe se verifique si las pérdidas al largo del estudio fueron semejantes entre los grupos. Análisis de la validez del estudio exige un poco más de experiencia del clínico y consulta la publicaciones en el área de epidemiología pueden contribuir para esta profundización.

En segundo lugar, la búsqueda de la mejor evidencia disponible presupone prioridad en las investigaciones en seres humanos, en especial a aquellas con desenlaces clínicos de significancia para el paciente y para la sociedad. Así, y conforme anteriormente descrito, ensayos clínicos en seres humanos y sobre desenlaces clínicamente relevantes (como caries, dolor o calidad de vida) son jerárquicamente superiores a ensayos laboratoriales o ensayos clínicos sobre des- 
enlaces intermediarios (como biofilm dental o conteo de unidades que forman colonias de microorganismos).

Considerando que aún no fueron publicadas revisiones sistemáticas o ensayos clínicos que responden a varias "preguntas" clínicas relevantes, en especial en la Odontología, se reconoce que las conductas clínicas podrán basarse en estudios con menor poder de evidencia científica.

Para contribuir en la evaluación crítica de artículos científicos, diferentes esquemas de evalua- ción crítica de artículos ya fueron propuestos, variando principalmente en consonancia con el diseño del estudio. La Figura 2 resume los aspectos principales a ser evaluados en los artículos seleccionados para lectura, con énfasis para la validez del estudio.

En especial, lectores de artículos científicos deben habituarse a evaluar potenciales sesgos, que son diferencias sistemáticas (que se repiten) entre dos grupos investigados (p.ej.: tratados y no tratados) o entre un grupo investigado y la

\begin{tabular}{|c|c|}
\hline $\begin{array}{l}\text { PREGUNTA } \\
\text { CIENTÍFICA }\end{array}$ & $\begin{array}{l}\text { Lea la introducción y el resumen. } \\
\text { - ¿Cuál el objetivo del estudio? } \\
\text { - ¿Cuál el cuadro teórico? } \\
\text { - ¿Cuáles los factores (exposiciones) en estudio? } \\
\text { - ¿Cuál el desenlace? Es clínicamente relevante? }\end{array}$ \\
\hline \multirow{2}{*}{$\begin{array}{l}\text { VALIDEZ INTERNA } \\
\qquad \begin{array}{l}\text { Y } \\
\text { ERRORES } \\
\text { SISTEMÁTICOS }\end{array}\end{array}$} & $\begin{array}{l}\text { Lea material y métodos. } \\
\text { - ¿Cuál es la población y el delineamiento de la investigación? } \\
\text { - ¿Hay potenciales sesgos de selección? } \\
\text { - ¿Hay potenciales sesgos de medición de las variables? }\end{array}$ \\
\hline & $\begin{array}{l}\text { Examine los resultados (figuras y tablas y, si necesario, el texto). } \\
\text { - ¿Cuáles son los hallazgos principales? } \\
\text { - ¿El potencial para confundir fue controlado? }\end{array}$ \\
\hline $\begin{array}{l}\text { INFERENCIA } \\
\text { ESTADÍSTICA }\end{array}$ & $\begin{array}{l}\text { - ¿Los resultados fueron estadísticamente significativos? } \\
\text { - ¿Cuál la magnitud del efecto? } \\
\text { - SI son significativos: ¿Cuál es el intervalo de confianza? } \\
\text { - Si no son significativos ¿Cuál el poder estadístico del estudio? } \\
\text { - ¿El tamaño de la muestra fue suficiente para detectar la diferen- } \\
\text { cia estimada? }\end{array}$ \\
\hline $\begin{array}{l}\text { SIGNIFICANCIA } \\
\text { CLÍNICA }\end{array}$ & - ¿El alcance y magnitud de los hallazgos tienen relevancia clínica? \\
\hline VALIDEZ EXTERNA & $\begin{array}{l}\text { - ¿Cuál es la capacidad de generalización de los resultados? } \\
\text { - ¿Son válidos para su contexto de trabajo? }\end{array}$ \\
\hline APLICABILIDAD & $\begin{array}{l}\text { - ¿Los resultados son aplicables en su contexto de trabajo? } \\
\text { - Sus conclusiones concuerdan con las conclusiones de los auto- } \\
\text { res? }\end{array}$ \\
\hline
\end{tabular}

Figura 2. Esquema de análisis crítico de artículos científicos (Adaptado de Schmidt \& Duncan, 2003.) 
población que debería representar. Su ocurrencia puede distorsionar los resultados presentados y llevar al lector y clínico a conclusiones equivocadas.

En general, errores sistemáticos son clasificados en sesgos de selección y de medición.

- Sesgos de selección distorsionan los resultados por la manera como los participantes son reclutados en el inicio del estudio o por la forma como son perdidos al largo del acompañamiento. Así, imagínese que en un estudio que compara dos fármacos (A versus $\mathrm{B}$ ) para reducción del dolor post- operatorio, la proporción de sujetos que retornan para la recolección de los datos es bien más pequeño en uno de los grupos (grupo A). Aunque los resultados indiquen que los pacientes de este grupo tengan menos dolor (e inclusive caractericen la diferencia como estadísticamente significante), es temerario concluir por su mayor eficacia. Es posible que la mayor proporción de individuos perdidos en este grupo esté relacionada a la empeora en el cuadro clínico o a efectos colaterales del fármaco, datos que son del desconocimiento de los lectores del artículo.

- Sesgos de medición (o de información) ocurren cuando las variables del estudio, sean ellas de exposición o de desenlace, son medidas erradamente. Así, en un estudio que evalúa la asociación entre el uso de biberón (exposición) y ocurrencia de caries dental (desenlace), pueden ocurrir errores por la forma como el dato referente a la práctica alimenticia es recolectada. Así, si el investigador pregunta sólo "Su hijo toma biberón?", el responsable puede entender que debe responder sólo sobre biberón con leche, omitiendo informaciones sobre jugos y tés ofrecidos en el biberón. De la misma forma, el examinador del desenlace puede hacer el examen físico con poca luminosidad y no detectar todas las lesiones de caries. Además de eso, puede no estar debidamente entrenado y confundir lesiones de caries con opacidad del esmalte. Un tipo especial de sesgo de medición muy común en estudios sobre caries en la infancia es el sesgo de memoria. En estudios transversales, es común del investigador preguntar sobre hábitos o comportamientos que ocurrieron hace muy tiempo, lo que puede llevar el responsable a una respuesta equivocada. En general, errores de medición ocurren con la misma intensidad en los grupos comparados y tienden a no detectar asociaciones que existen en el mundo real.

Investigaciones también pueden resultar en conclusiones equivocadas debido a "factores de confusión", también designados como sesgos de confusión. Conforme ya fue ilustrado, este tipo de error ocurre cuando por detrás de una variable que produce un resultado, está "escondida" otra variable ("factor de confusión"), que en la verdad es la responsable por el desfecho. Es fundamental que el investigador recolecte los datos sobre todas las variables potencialmente confundidoras para que, en el análisis de los resultados, pueda controlarlas con técnicas estadísticas. Así, técnicas de análisis multivariables pueden "remover" el efecto de factores de confusión.

Además de errores sistemáticos, lectores de artículo deben quedar atentos a la posibilidad de errores aleatorios. Eso ocurre en dos situaciones: cuando un estudio afirma existir una asociación que en la verdad no existe en la población (llamado de error alfa) o cuando el estudio no consigue detectar una asociación que efectivamente existe (error beta). Es importante comprender 
que ambas situaciones no ocurrieron por selección inadecuada de participantes o por error en las medidas, y sí porque el azar, difirió la muestra de la población. Aunque no sea propósito de esta publicación expandir la discusión a respeto, es importante el clínico comprender que estudios con pequeño número de muestras presentan una gran posibilidad de incidir en error beta, o sea, no detectar asociaciones que existen en el mundo real. Un ejemplo trivial en este sentido es cuando estudios concluyen que una técnica A es "tan eficaz" en cuanto a técnica B a partir de la evaluación de los resultados en un número insuficiente de pacientes.

Por fin, y de interés creciente para el lector de artículos científicos, es importante comprender cuando los resultados de artículos son mostrados en la forma de "intervalos de confianza". Teniendo como un hecho que un estudio no presenta sesgos que modifiquen los resultados, el intervalo de confianza 95\% representa, con un margen de error del 5\%, el rango de variación del resultado en la POBLACIÓN, y no el de la MUESTRA del estudio. Así, cuando una investigación que evalúa caries en la infancia describe la Razón de prevalencia de consumir dulces entre horas es 1,5, con un intervalo de confianza 95\% es de 1,4-1,6, la interpretación debe ser: la probabilidad de que un niño presente caries en la infancia si consume dulces entre las comidas es 50\% mayor (en la muestra). En la población, el aumento en la probabilidad de que tales niños presenten caries (con un margen de error del 5\%) varía entre $40 \%$ a $60 \%$.

\section{Aplicación de la evidencia para la toma de decisiones en la práctica clínica}

Después de reunir la mejor evidencia disponible para responder a una determinada pregunta y analizar su validez, se sigue el análisis en cuanto a la aplicación de la evidencia en la toma de decisiones. En la actualidad, viene siendo crecientemente valorado el concepto de una "toma de decisión compartida" entre profesionales de la salud y pacientes (17). En este sentido, profesionales de la salud contribuyen con el cuerpo de evidencias de investigaciones, incluyendo el peso de los beneficios y de los riesgos, y los pacientes manifiestan lo que desean en relación a su cuidado, incluyendo los riesgos a que desean someterse. De esta forma, la toma de decisiones debe basarse en evidencias científicas sólidas y en las preferencias de los pacientes.

Teniendo por base el presupuesto arriba, otras cuestiones deben ser consideradas. Aunque se desee que la comprobación de decisiones clínicas se base en evidencia de nivel I, frecuentemente eso no es posible, no habiendo respuesta con alto grado de confianza a un gran número de preguntas clínicas. Si por un lado este hecho demuestra la complejidad y las limitaciones de basar la práctica clínica en evidencias, por otro permite al menos que se estime el grado de certeza que envuelve cada decisión clínica, conocimiento que puede ser compartido con el paciente.

La disponibilidad, costo y la facilidad de la aplicación de pruebas diagnósticos o procedimientos preventivos y terapéuticos es otro factor que puede influenciar en la toma de decisiones. Es posible que la "mejor decisión para aquel paciente" no sea exactamente aquella con mayor grado de evidencia demostrada. En este sentido, es fundamental el conocimiento y capacidad de juicio crítico y objetivo del profesional en relación la alternativas disponibles.

Conforme anteriormente abordado, diferentes asociaciones y entidades han desarrollado di- 
rectrices para responder a las dudas clínicas, lo que contribuye con el clínico que no dispone de tiempo para un análisis crítico de la literatura disponible. La calidad de tales directrices y el grado con que contribuyen para la práctica basada en evidencias tiene crecientemente aumentado, una vez que ellas tienen explícito el nivel de evidencia en que cada recomendación se basa. Aún directrices con alto grado de detalle no prescinden, sin embargo, de una evaluación específica en relación su aplicabilidad en el contexto local, principalmente se fueron producidas por investigadores de otro país.

En la Odontopediatría, un ejemplo de práctica clínica basada en evidencias es la utilización de selladores de fosas y fisuras (18). La síntesis de la directriz desarrollada por la Asociación Brasileña de Odontopediatria afirma que Selladores de fosas y fisuras deben ser aplicados y monitoreados en niños y adolescentes con mayor vulnerabilidad a la enfermedad caries, por su comprobada efectividad en la reducción de la incidencia de lesiones en fosas y fisuras [evidencia de nivel A - basada en revisiones sistemáticas y ensayos clínicos randomizados], reducción en la necesidad de futuro tratamiento restaurador [evidencia de nivel B - basada en estudios de cohorte] y más pequeños costes para individuos e instituciones [evidencia de nivel A].

\section{Consideraciones finales}

La literatura viene crecientemente demostrando que las decisiones clínicas de los profesionales de la salud, principalmente aquellas relacionadas a la prevención y tratamiento de los diferentes afecciones, deben basarse en la mejor evidencia disponible. En ese sentido, investigadores en el área de Odontopediatría deben desarrollar proyectos que efectivamente contribuyan con una mejora en el nivel de evidencias de forma a responder cuestiones clínicamente relevantes. A la vez, es fundamental que los clínicos asuman su papel como agentes de una práctica basada en la mejor evidencia posible. Eso parece esencial para que la Odontopediatría cumpla de forma realiza su papel de promover la salud del niño.

\section{Referencias}

1. Marinho VCC. Prática Odontológica Baseada em Evidência - Sua Aplicação na Promoção de Saúde Bucal. In: Buischi YP Promoção de Saúde Bucal na Clínica Odontológica. 1. ed. São Paulo: Artes Médicas; 2000. p. 339-359.

2. Sackett DL, Straus SE, Richardson WS, Rosenberg W, Haynes RB. Medicina baseada em evidências - Prática e ensino., 2a. ed. Porto Alegre: Artmed, 2003.

3. Schmidt MI; Duncan BB. Epidemiologia Clínica e Medicina Baseada em Evidências. In: Rouquayrol MZ e de Almeida Filho N. Epidemiologia e Saúde. 6a. ed. Rio de Janeiro: Medsi, , 2003.

4. Rosenfeld M, Seferiadis A, Gunnarsson R. Active involvement and intervention in patients exposed to whiplash trauma in automobile crashes reduces costs: a randomized, controlled clinical trial and health economic evaluation. Spine. 2006;31:1799-804.

5. Echt DS, Liebson PR, Mitchell LB et al. Mortality and morbidity in patients receiving encainide, flecainide, or placebo. The Cardiac Arrhythmia Suppression Trial. N Engl J Med. 1991;324:781-8.

6. Kay EJ, Locker D. Is dental health education effective? A systematic review of current evidence. Community Dent Health. 1996; 24:231-5.

7. Hamilton FA, Davis KE, Blinkhorn AS. An oral health promotion programme for nursing caries. Int J Paediatr Dent. 1999; 9:195-200. 
8. Petersen PE, Peng B, Tai B, Bian Z, Fan M. Effect of a school-based oral health education programme in Wuhan City, Peoples Republic of China. Int Dent J. 2004;54:33-41.

9. Vachirarojpisan T, Shinada K, Kawaguchi Y. The process and outcome of a programme for preventing early childhood caries in Thailand. Community Dent Health. 2005; 22:253-259.

10. Rossouw JE, Anderson GL, Prentice RL et al. Writing Group for the Women's Health Initiative Investigators. Risks and benefits of estrogen plus progestin in healthy postmenopausal women: principal results From the Women's Health Initiative randomized controlled trial. JAMA. 2002;288:321-33.

11. Feldens CA, Giugliani ER, Duncan BB, Drachler Mde L, Vítolo MR. Effect of a nutritional intervention on childhood caries: reply to Seow. J Evid Based Dent Pract. 2011;11:164-5.

12. Howes N, Chagla L, Thorpe M et al. Surgical practice is evidence based. Br J Surg, 84:1220-1223,1997.

13. Butani Y, Levy SM, Nowak AJ et al. Overview of the evidence for clinical interventions in pediatric dentistry. Pediatr Dent. 2005;27:6-11.

14. Nobre MR, Bernardo WM, Jatene FB. A prática clínica baseada em evidencias. Parte I - Questões clínicas bem construídas. Rev Assoc Med Bras 2003; 49:445-9.

15. Bernardo WM, Nobre MR, Jatene FB. A prática clínica baseada em evidências: parte II - buscando as evidências em fontes de informação. Rev Assoc Med Bras. 2004;50:104-8.

16. Phillips B, Ball C, Sackett D et al. Oxford-Centre for Evidence Based Medicine. Centre for Evidence Based Medicine. Acessado (2012 Mar 22). Disponível em: URL: http: / / www.cebm.net/.

17. Fletcher RH, Fletcher, SW. Epidemiologia Clínica - Elementos Essenciais. 4a . Ed. Porto Alegre: Artmed; 2006.

18. Feldens CA, Hebling J, Santos-Pinto LAM, Kramer PF. Diretriz sobre políticas no uso de selantes. In: Massara MLA; Rédua PCB (coord.). Manual de Referência para Procedimentos Clínicos em Odontopediatria. São Paulo: Ed. Santos; 2009.

Recibido: 04-04-12

Aceptado: 19-04-12

Correspondencia: cafeldens@terra.com.br

Texto Traducido por: Fabian Calixto Fraiz,José Miguel Amenábar Céspedes, Guido Perona Miguel de Priego 